\title{
Short vs. Long Spinal Cord T2 Lesions: Differential Diagnosis
}

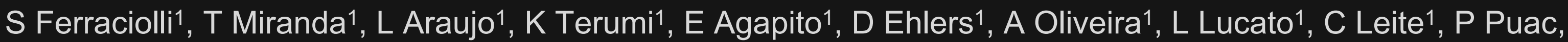

C Zamora2, M Castillo2 ${ }^{1}$ InRad - HC- FMUSP, São Paulo, Brazil, ${ }^{2}$ UNC, Chapel Hill, NC, USA

\section{Purpose}

To review imaging findings \& propose a step-by-step approach to generate a differential diagnosis of short \& long T2 hyperintense spinal cord (SC) lesions.

\section{Approach/Methods}

We searched teaching files of 2 institutions for short \& long T2 bright SC lesions \& review \& discuss pertinent literature emphasizing distinguishing features for these entities.

\section{Findings/Discussion}

T2 hyperintense SC lesions have a long differential diagnosis including MRI artifacts; trauma; compression myelopathy; 1ry \& 2ry neoplasms; radiation myelitis; inflammatory \& necrotizing diseases; subacute combined degeneration (B12 deficiency); drug toxicity; vascular causes; infections, post infective and granulomatous diseases \& cavitary lesions among less common causes. Correlation between clinical data \& imaging characteristics of T2 hyperintense SC lesions helps us generate a deferential diagnosis to suggest the correct diagnosis.

A practical approach is a time-based scheme which emphasizes acute, chronic \& relapsing symptoms. As shown in the Table, multiple diagnoses overlap in the same time classification so the first step is to differentiate between short \& long T2 lesions followed by lesion location on the axial T2 images which helps differentiate between lesions involving either of white \& gray matter such as infarcts, polio and Hirayama disease in the anterior horns; B12 deficiency, copper deficiency, syphilis \& less frequently infarcts in posterior columns; \& acute transverse myelitis, inflammation (ADEM, NMO and some anti-MOG + myelitis) \& ependymoma which occur in the central SC.
Presence of other signs such as the pancake sign in compressive myelopathy, trident sign in sarcoidosis, owl/snake eyes in anterior spinal artery infarct, inverted $V$ sign in B12 deficiency \& bright spots in neuromyelitis optica as shown below.

\section{Summary/Conclusion}

In this educational exhibit we describe short \& long T2 hyperintense lesions, highlight the features that suggest the correct diagnosis, provide a short literature review as well as a diagnostic approach.

\begin{tabular}{|c|c|c|c|}
\hline Onset & $\begin{array}{c}\text { MRI } \\
\text { Sagittal }\end{array}$ & $\begin{array}{l}\text { Differential } \\
\text { Diagnosis }\end{array}$ & Imaging Findings \\
\hline \multirow[t]{2}{*}{$\begin{array}{l}\text { ACUTE/ } \\
\text { SUBACUTE }\end{array}$} & SHORT & $\begin{array}{l}\text { MS } \\
\text { Infective } \\
\text { Autoimmune } \\
\text { Sarcoidosis }\end{array}$ & $\begin{array}{l}\text { Viral: central grey matter } \\
\text { Tuberculosis: WM nodules or target } \\
\text { lesions } \\
\text { Parasitic: Eccentric nodular pattern }\end{array}$ \\
\hline & LONG & $\begin{array}{l}\text { NMOSD } \\
\text { Autoimmune } \\
\text { ADEM } \\
\text { Sarcoidosis } \\
\text { Infective (viral) } \\
\text { Paraneoplastic } \\
\text { Vascular } \\
\text { Metabolic }\end{array}$ & $\begin{array}{l}\text { NMOSD: Central grey } \\
\text { matter/transverse myelitis } \\
\text { Sarcoidosis: Variable: dorsal cord } \\
\text { subpial GAD enhancement may help } \\
\text { differentiate from NMOSD } \\
\text { Metabolic: Symmetric T2 } \\
\text { hyperintensity dorsal tracts }\end{array}$ \\
\hline \multirow[t]{2}{*}{$\begin{array}{l}\text { CHRONIC } \\
\text { PROGRESSIVE }\end{array}$} & SHORT & $\begin{array}{l}\text { Sarcoidosis } \\
\text { MS } \\
\text { Neoplastic } \\
\text { (ependymoma, } \\
\text { astrocytoma, } \\
\text { metastatic) }\end{array}$ & $\begin{array}{l}\text { Ependymoma: homogenous } \\
\text { enhancement; clear borders; central; } \\
\text { rostral and caudal cysts } \\
\text { Astrocytoma: heterogeneous or no } \\
\text { enhancement; irregular margins; } \\
\text { eccentric location } \\
\text { Metastatic: eccentric location }\end{array}$ \\
\hline & LONG & $\begin{array}{l}\text { Paraneoplastic } \\
\text { Sarcoidosis } \\
\text { Chronic } \\
\text { infection } \\
\text { Vascular (MF) } \\
\text { Metabolic } \\
\text { Neoplastic }\end{array}$ & $\begin{array}{l}\text { Syphilis and HIV: dorsal column (may } \\
\text { mimic metabolic) } \\
\text { HTLV-1: lateral columns > central and } \\
\text { anterior cord } \\
\text { Astrocytoma > Ependymoma }\end{array}$ \\
\hline \multirow[t]{2}{*}{ RELAPSING } & SHORT & MS & $\begin{array}{l}\text { Posterolateral lesions; less than half } \\
\text { the axial area }\end{array}$ \\
\hline & LONG & $\begin{array}{l}\text { NMOSD } \\
\text { Sarcoidosis } \\
\text { Vascular }\end{array}$ & \\
\hline
\end{tabular}



Left to right: SHORT lesions. 1. Posterior disc herniation causes compressive myelopathy with high T2 signal. Post-operative T1WI Gd+ shows classic "pancake" sign; 2. Screwdriver puncture lesion shows short T2 bright area with posterolateral blooming artifact; 3 . Small cystic lesion of neurocysticercosis with high T2 in SC; 4 . Renal cell carcinoma metastasis seen as nodular lesion with heterogeneous T2 signal \& hemorrhage with intense contrast enhancement; 5 . Sarcoidosis with high T2 signal due to edema showing patchy contrast enhancement and in axial view the "trident" sign; 6. MS lesions with short longitudinal extension \& high T2 signal. LONG lesions: 7. ADEM with long, tumefactive, T2 bright lesion involving the cervical SC; 8 . Infarct in anterior spinal artery territory shows the characteristic owl eyes sign; 9. HIV-related myelopathy shows atrophy \& longitudinally extensive high T2 signal in anterior gray matter; 10. B12-deficiency with long T2 hyperintensity in posterior funiculi with typical inverted V sign; 11. Astrocytoma characterized by long/tumefactive and heterogeneous T2 hyperintense lesion with a small cyst; 12 . NMOSD shows longitudinally extensive lesion with high T2 signal including area postrema \& intralesional bright spots. 